\title{
CDISC SEND Non-Neoplastic Finding Type Terminology
}

National Cancer Institute

\section{Source}

National Cancer Institute. CDISC SEND Non-Neoplastic Finding Type Terminology. NCI Thesaurus. Code C120531.

Terminology associated with the non-neoplastic finding type codelist of the Clinical Data Interchange Standards Consortium (CDISC) Standard for the Exchange of Non-clinical Data (SEND). 